\title{
Performance Analysis of Ultra-Wideband Channel for Short-Range Monopulse Radar at Ka-Band
}

\author{
Naohiko Iwakiri, ${ }^{1}$ Natsuki Hashimoto, ${ }^{2}$ and Takehiko Kobayashi ${ }^{2}$ \\ ${ }^{1}$ Nano-Satellite Center, The University of Tokyo, Tokyo 113-8656, Japan \\ ${ }^{2}$ Wireless Systems Laboratory, Tokyo Denki University, Tokyo 101-8457, Japan \\ Correspondence should be addressed to Naohiko Iwakiri, iwaikiri@nsat.t.u-tokyo.ac.jp
}

Received 28 October 2011; Accepted 2 February 2012

Academic Editor: Hsiao-Chin Chen

Copyright (C) 2012 Naohiko Iwakiri et al. This is an open access article distributed under the Creative Commons Attribution License, which permits unrestricted use, distribution, and reproduction in any medium, provided the original work is properly cited.

\begin{abstract}
High-range resolution is inherently provided with Ka-band ultra-wideband (UWB) vehicular radars. The authors have developed a prototype UWB monopulse radar equipped with a two-element receiving antenna array and reported its measurement results. In this paper, a more detailed verification using these measurements is presented. The measurements were analyzed employing matched filtering and eigendecomposition, and then multipath components were extracted to examine the behavior of received UWB monopulse signals. Next, conventional direction finding algorithms based on narrowband assumption were evaluated using the extracted multipath components, resulting in acceptable angle-of-arrival (AOA) from the UWB monopulse signal regardless of wideband signals. Performance degradation due to a number of averaging the received monopulses was also examined to design suitable radar's waveforms.
\end{abstract}

\section{Introduction}

Ultra-wideband (UWB) vehicular radars, recently approved by governments of many counties, are expected to be instrumental in reducing traffic accidents [1]. In comparison with conventional vehicular sensing systems using ultrasound, visible and infra-red light, and millimeter waves, Ka-band UWB radar systems hold several advantages, such as no blind angle [2], high-resolution in ranging, weatherproof, and lower price than millimeter-wave radars. Since transmission power of UWB systems is restricted at a level low enough to avoid interference with conventional narrowband systems, UWB vehicular radars are used at short range for collision warning, blind spot detection, parking aid, and precrash control.

During the last decade, there has been much progress on UWB technologies. To understand the behavior of UWB signals, the UWB propagation channel is measured and characterized as described in [3]. Different channel models for practical UWB systems have been proposed in multipath environments [4-6]. Since UWB signals potentially have high resolution in ranging, the different UWB ranging systems, in multipath environments, have been investigated in $[7,8]$.

The UWB signals for the vehicular radar have also been measured and modeled in some ways: a generic stochastic uniformly tapped delay line model of multipath UWB channel [9], outdoor channel modeling based on measurements by means of a vector network analyzer $[10,11]$, and a pulse-based UWB sensor [12]. However, it is insufficient to characterize the behavior of UWB channel signals for shortrange monopulse radar at Ka-band, since these works focus on generic modeling below Ka-band. Ranging and direction finding are a key feature of UWB radar systems. Thus, we need to understand these characteristics for Ka-band using a practical analysis method based on measurements.

The authors have developed a prototype UWB monopulse radar equipped with a receive two-element antenna array. The performances of ranging and direction finding by delta-over-sigma (DOS) scheme were evaluated using measurements obtained in a radio anechoic chamber and outdoor environments [13]. However, several problems arise when analyzing the performance of this radar setup. First, the DOS scheme uses sum and differential signals impinging 


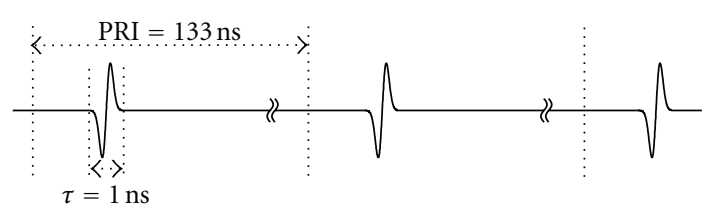

FIgURE 1: Train of transmitted monopulses.

on the two-element array. A predetermined broadside is required for the DOS scheme to estimate angle-of-arrival (AOA) even if the radar moves toward different directions. Moreover, the DOS scheme specialized to a two-element array makes it impractical as an implementation for general multielement antenna array.

Since the measurements were conducted with independent reception for DOA scheme at each of the two-element array, the prototype UWB monopulse radar could not be collected enough of received signals. We, therefore, developed an analysis technique to model UWB monopulse signals by focusing on two ways to understand characteristics of ranging and direction finding, regardless of some constraints. First, the measurements were analyzed to characterize the behavior of multipath components from a target contained in the received signals. Next, instead of applying the DOS scheme, conventional techniques of pulse compression for ranging and multiple signal classification (MUSIC) or minimum variance distortionless response (MVDR) algorithms for direction finding were adopted to evaluate the applicability in UWB vehicular radars. The obtained results provide insight into UWB monopulse radar design.

The rest of this paper is organized as follows: modeling multipath components is discussed in Section 2. Experimental verification of the prototype radar from measurements in a radio anechoic chamber is described in Section 3. Performance analysis of the UWB monopulse signals in outdoor environments is presented in Section 4. Finally, the conclusions are drawn in Section 5.

\section{Modeling Multipath Components}

The UWB monopulse radar can resolve more multipath components than conventional narrowband radar systems because of its high resolution in ranging. Moreover, the AOA of each of the components is obtained if multiple-element antennas are adopted and conventional direction finding algorithms are available. However, an optimum analysis scheme for the received signal is required to design reliable UWB radar systems with acceptable performance. We then derive the detection scheme for resolvable or nonresolvable multipath components within received UWB monopulse signals.

2.1. Resolvable Multipath Components. A train of transmitted monopulses demonstrated in this work is shown in Figure 1. There is a single monopulse in each pulse repetition interval (PRI). The signal impinging on the target and the reflected signal observed by the receive array are assumed to be plane waves in a static channel. If $M$ signals impinge on the $L$-element array from distinct AOAs, the received signal commonly used in array processing is given by [14]

$$
\mathbf{x}(t)=\mathbf{A}(\boldsymbol{\theta}) \mathbf{s}(t)+\mathbf{n}(t)
$$

where a steering matrix $\mathbf{A}(\boldsymbol{\theta})$ and a vector of signal waveforms $\mathbf{s}(t)$ are defined by

$$
\begin{aligned}
\mathbf{A}(\boldsymbol{\theta}) & =\left[\mathbf{a}\left(\boldsymbol{\theta}_{1}\right), \ldots, \mathbf{a}\left(\boldsymbol{\theta}_{M}\right)\right], \\
\mathbf{s}(t) & =\left[s(t), \ldots, s_{M}(t)\right]^{T} .
\end{aligned}
$$

Assuming a uniform linear array (ULA) having interelement spacing of $d$, a steering vector $\mathbf{a}\left(\theta_{m}\right)$ can be written by

$$
\mathbf{a}\left(\theta_{m}\right)=\left[a_{1}\left(\theta_{m}\right), \ldots, a_{L}\left(\theta_{m}\right)\right]^{T}, \quad m=1, \ldots, M .
$$

For the plane wave case, $l$-th elements at the AOA $\theta_{m}$ are given by

$$
a_{l}\left(\theta_{m}\right)=e^{-j(l-1)(2 \pi / \lambda) d \sin \theta_{m}}, \quad l=1, \ldots, L,
$$

where $\lambda$ is wavelength.

The received signals are assumed as discrete signals, and then the received signal matrix per PRI can be written as

$$
\mathbf{X}=\left[\mathbf{x}(1), \ldots, \mathbf{x}\left(N_{\text {pri }}\right)\right]
$$

where $N_{\text {pri }}$ is a number of signal samples per PRI. A pulse compression can be carried out for each element output to detect multipath components passed through a matched filter. This output of $l$-th element is simply the inner product between received signals of $l$-th element and replica signals, namely, pulse compression [15], as given by

$$
r_{l}(n)=\mathbf{X}_{l}\left(t_{n}\right) \cdot \mathbf{h},
$$

where $t_{n}=n+1, n+2, \ldots, n+N_{\text {pri }}-N_{\text {seq }}, n=0,1, \ldots, N_{\text {pri }}-$ $N_{\text {seq }}$, and $\mathbf{h}$ is a complex conjugate column vector of the replica signal with length of $N_{\text {rep }}$. Thus, all of the $N_{\text {pri }}-N_{\text {seq }}+1$ outputs are obtained for each PRI, and then the outputs of the $l$-th element are averaged over $N_{\text {ave }}$ PRIs:

$$
\bar{r}_{l}(n)=\frac{1}{N_{\text {ave }}} \sum_{a=1}^{N_{\text {ave }}} r_{l, a}(n) .
$$

Finally, $k$ th multipath components can be extracted to choose the signal strength higher than the optimum threshold $\gamma$, as given by

$$
\left|\bar{r}_{l}\left(n_{k}\right)\right|>\gamma, \quad k=1,2, \ldots, K,
$$

where $n_{k}$ is a delay sample from the PRI boundary of the $k$-th multipath components. Therefore, for a static environment, the total of $K$ peaks can be related to the resolvable multipath components. 


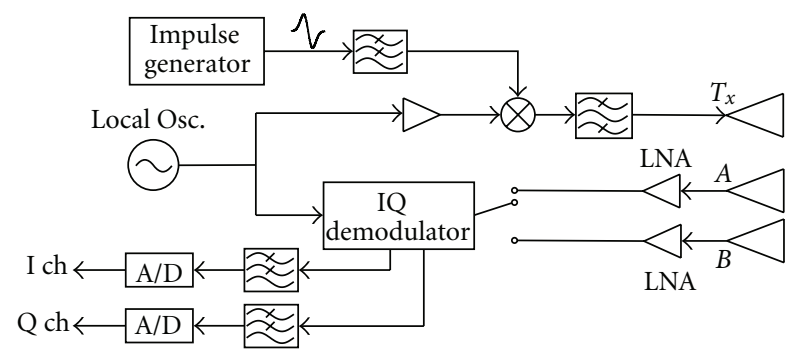

FIgURE 2: The block diagram of the prototype radar transmitterreceiver.

2.2. Nonresolvable Multipath Components. We next consider how to detect nonresolvable multipath components. The sample covariance matrix is calculated using $2 N_{\mathrm{smp}}+1$ samples around $k$-th resolvable multipath components:

$$
\mathbf{R}_{k}=\frac{1}{2 N_{\mathrm{smp}}+1} \mathbf{X}\left(n_{i}\right) \mathbf{X}^{H}\left(n_{i}\right),
$$

where $n_{i}=n_{k}-N_{\mathrm{smp}}, n_{k}-N_{\mathrm{smp}}+1, \ldots, n_{k}, \ldots, n_{k}+N_{\mathrm{smp}}$.

Up to this point, an arbitrary number of array elements have been discussed. In subsequent discussions, we focus on an $L=2$ case since a two-element array was implemented in our prototype radar system. Two eigenvalues $\lambda_{1}$ and $\lambda_{2}$ can be obtained from eigendecomposition of $\mathbf{R}_{k}$, because $\mathbf{R}_{k}$ is guaranteed positive definite due to Hermitian matrix. There are three types of eigenvalue distribution: $\lambda_{1} \geq \lambda_{2}>\sigma^{2}$ when two or more signals having different AOAs impinging on the receive array, or a single AOA cannot correctly resolve owing to inadequate accuracy of the elements in $\mathbf{R}_{k}$, where $\sigma^{2}$ is a noise variance; $\lambda_{1}>\lambda_{2}=\sigma^{2}$ when a single signal impinges on the receive array; $\lambda_{1}=\lambda_{2}=\sigma^{2}$ when none of signals is received.

Nonresolvable multipath components within each multipath component will be estimated from this classification because of the Nyquist rate. The basic idea is to find a criterion of nonresolvable multipath components from measurements collected in a single-path environment realized in radio anechoic chamber. The criterion can be obtained to apply the second type of eigenvalue distribution. Specifically, the nonresolvable multipath component can be estimated if $\lambda_{2}$ is larger than the boundary, since a boundary between resolvable and nonresolvable multipath components is determined from the distribution of $\lambda_{2}$. Hence, the behavior of a multipath component in outdoor environments is characterized when using a passenger vehicle as a target. This criterion can be easily extended to an array having elements more than 2 .

\section{Anechoic Chamber Experiments}

3.1. Experimental Setup. The block diagram of a prototype Ka-band UWB pulse radar is depicted in Figure 2, which is the same system as our previously published work [13], except that the received signals were directly input to an IQ demodulator without passing through a coupler. The experiment was set for a single-path environment in a
TABLE 1: Specification of experiments.

\begin{tabular}{lc}
\hline Waveform & Modulated Gaussian monopulse \\
\hline $\begin{array}{l}\text { Pulse duration } \\
\text { Frequency } \\
\text { bandwidth }\end{array}$ & $1 \mathrm{~ns}$ \\
Sampling rate & 10 or $20 \mathrm{Gsps}$ \\
Antennas & Slot antennas \\
Polarization & Vertical-vertical \\
Element spacing & $5.4 \mathrm{~mm}$ \\
Spectrum density & $<-44 \mathrm{dBm} / \mathrm{MHz}$ \\
of average emission &
\end{tabular}

radio anechoic chamber. The measurements were analyzed to obtain parameters required to characterize resolvable or nonresolvable UWB monopulse signals in outdoor environments. Gaussian monopulse has been widely adopted for UWB systems to satisfy FCC spectrum mask regulations [16]. Various practical monopulse design techniques have been developed in [17-19]. The performance of these design techniques has also been analyzed in different ways: principle of space-time array processing [20], target recognition reflected on different obstacles [21], and position estimation [22]. We also adopted Gaussian monopulse due to its ease of use with our analysis methods. An arbitrary waveform generator with a sampling rate of $25 \mathrm{GHz}$ was used as an impulse generator.

In the first experiment, a trihedral corner reflector was used as a target, comprising three right-angled isosceles triangles with $210 \mathrm{~mm}$ long sides. Its radar cross section was approximately $191 \mathrm{~m}^{2}$. The reflector was placed at a distance of $2.8 \mathrm{~m}$ from the radar. The radar was rotated from $-30^{\circ}$ to $30^{\circ}$ in intervals of $1^{\circ}$, and then received signals were recorded. A direction of $0^{\circ}$ was defined as broadside of the two receive antennas. Transmit and receive antennas were slot antennas with vertical polarization. The impulse generator outputs baseband Gaussian monopulse trains with monopulse duration of $1 \mathrm{~ns}$ occupying a bandwidth of $2 \mathrm{GHz}$, as shown in Figure 3. The trains are upconverted through RF modules by a carrier frequency of $26.5 \mathrm{GHz}$ and emitted from the transmitting antenna. The spectrum density of the average emission in this band was selected less than $-44 \mathrm{dBm} / \mathrm{MHz}$ to satisfy FCC regulations [1]. Each received signal impinging on the two-element array is down-converted to baseband by the IQ demodulator, analogto-digital converted at a sampling rate of 10 or $20 \mathrm{Gsps}$, and then recorded in a digital storage oscilloscope (DSO). The transmitted and received UWB monopulse signals are shown in Figures 3(a) and 3(b), respectively. The latter is distorted due to mismatching in the RF components. Major parameters of the experiment are listed in Table 1.

The above setup is capable of collecting data of the single path impinging on a horizontally placed two-element array with half-wavelength element spacing. The received signal performance and the results for analyzing the measurements are described in the next section. 


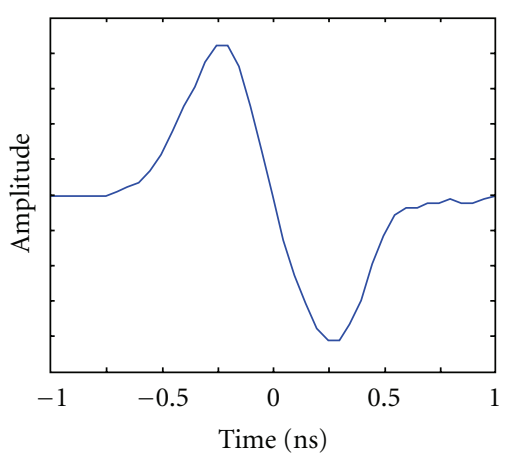

(a) Transmitted

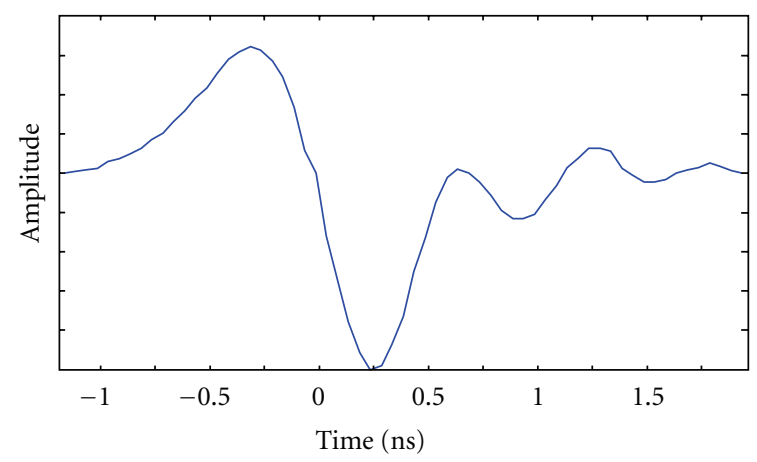

(b) Received

FIgURE 3: Baseband monopulse waveforms.

A received signal power typically attenuates as the distance between the radar and the target increases. Hence, resolution accuracy of ranging and direction finding will depend on the received power. A technique that averages received monopulses is useful to increase received signal gain without increasing transmit power. We then estimated the resolution accuracy by evaluating the different numbers of averaging monopulses at the receiver. The result is discussed in Section 4.5.

3.2. Performance Analysis. Received multipath components, after averaging over $10^{4}$ PRIs, were extracted by pulse compression, as given by (7). The reference signals of the matched filter used for both the $I$ and $Q$ channels were the received monopulse signal shown in Figure 3(b). The pulsecompressed output when the radar is directed to $0^{\circ}$ is shown in Figure 4, where a single sharp peak is observed. The plot shows that the number of averaging $10^{4} \mathrm{PRI}$ is sufficient to extract a single peak from the noisy received signals, and the outputs directed to the other angles also have a single peak. However, since the antenna pattern is not isotropic, the peak strength decreases as the direction moves away from broadside. The peak strength from $-30^{\circ}$ to $30^{\circ}$ is shown in Figure 5.

Next, the covariance matrix $\mathbf{R}$ and its eigenvalues were calculated for each extracted peak, as described in Section 2.2. To collect more samples around the peak, $N_{\text {smp }}=2$ (total 5 samples) was selected for 20 Gsps sampling. The obtained 61 pairs of eigenvalues $\lambda_{1}$ and $\lambda_{2}$ were then normalized by $\lambda_{1}$. The eigenvalue distribution shown in Figure 6 revealed that all the $\lambda_{2}$ are sufficiently smaller than unity regardless of direction. It is thus concluded that $\lambda_{1}$ is a signal or resolvable component and $\lambda_{2}$ is a noise or nonresolvable component, and its boundary is $10^{-1}$. This boundary will be used as the criterion of detection of nonresolvable multipath components in the outdoor experiments, as described in Section 4.2.

Finally, the AOA was estimated for each of the extracted peaks using MUSIC algorithm [14] to verify its applicability to the UWB monopulse signals. The obtained AOAs are plotted in Figure 7. The two regression curves corresponding

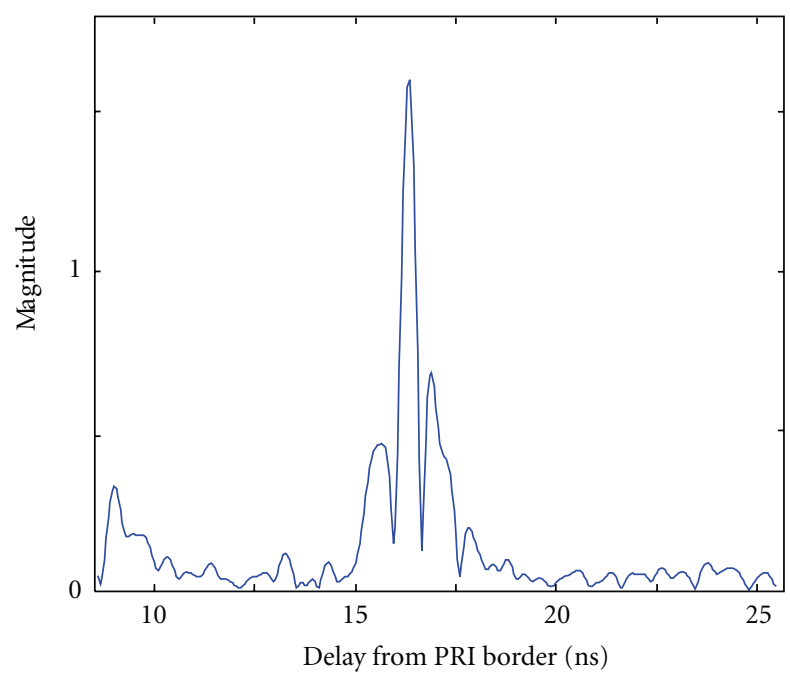

FIGURE 4: Pulse-compressed output signals from broadside in anechoic chamber experiments.

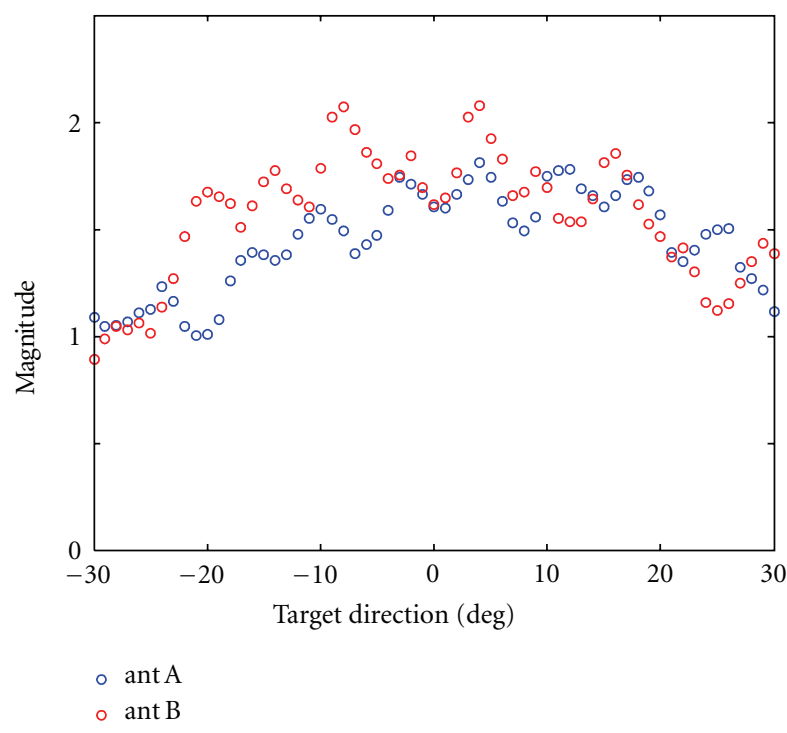

Figure 5: Magnitude of extracted multipath components corresponding to different angles in anechoic chamber experiments. 


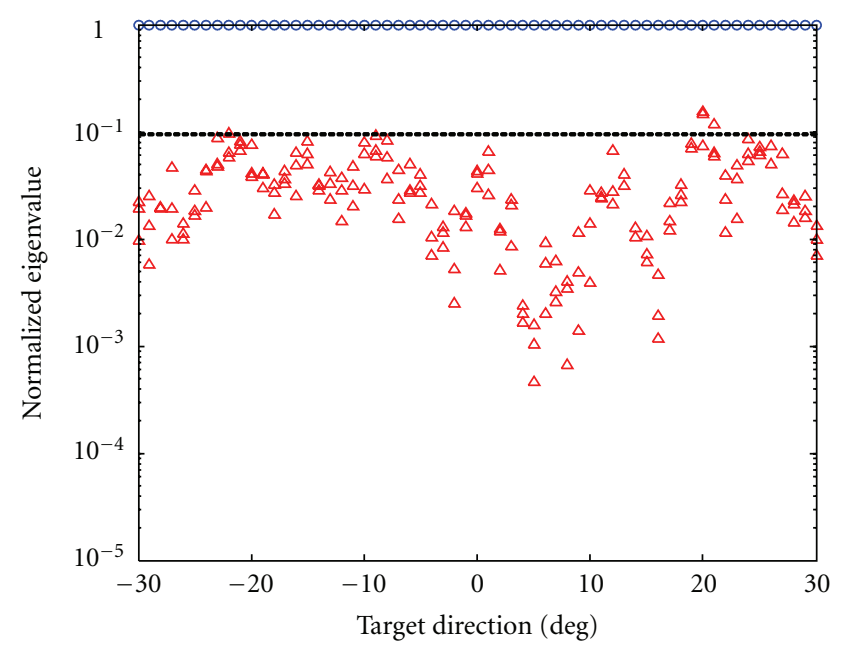

- 1st eigenvalue

$\triangle$ 2nd eigenvalue

Figure 6: Normalized eigenvalue distribution derived from the measurements in anechoic chamber experiments.

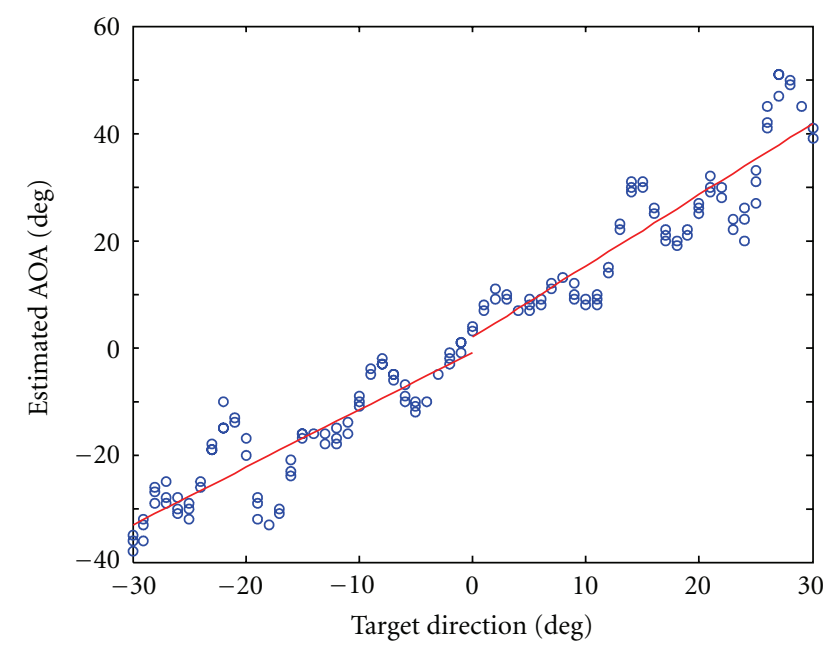

FIGURE 7: Estimated AOA by MUSIC derived from the measurements in anechoic chamber experiments.

to plus and minus angles are also plotted by solid lines. The plot shows that the MUSIC algorithm can be utilized for UWB signal with monopulse duration of $1 \mathrm{~ns}$ and carrier frequency of $26 \mathrm{GHz}$. This result can prove that the carrier-to-baseband frequency ratio of 26 is sufficient for the conventional direction finding algorithm regardless of the UWB signals.

\section{Outdoor Experiments}

4.1. Experimental Setup. The next experiment was conducted in outdoor environments. A four-door sedan, $4.73 \mathrm{~m}$ long, $1.80 \mathrm{~m}$ wide, and $1.44 \mathrm{~m}$ high, was used as a radar target in the outdoor experiment. The vehicle, parked on a broadside, is shown in Figure 8. The different locations

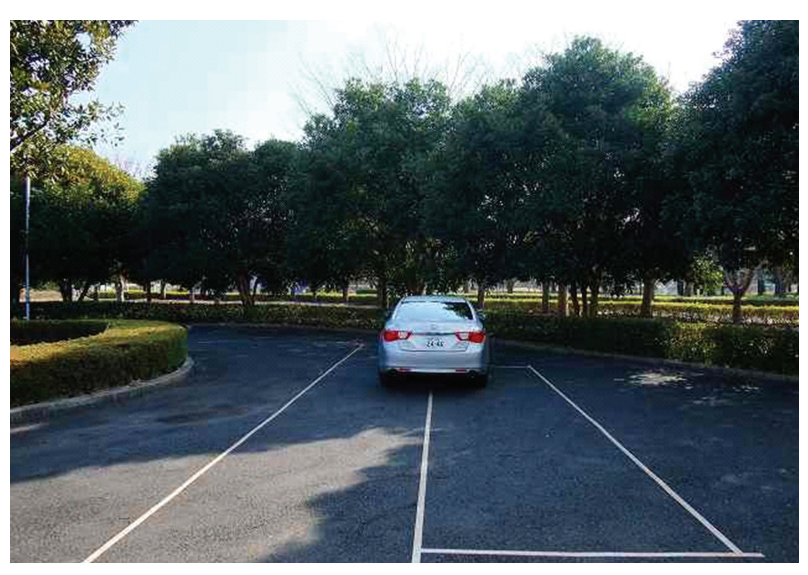

FIgURE 8: A radar target on a broadside in outdoor experiments.

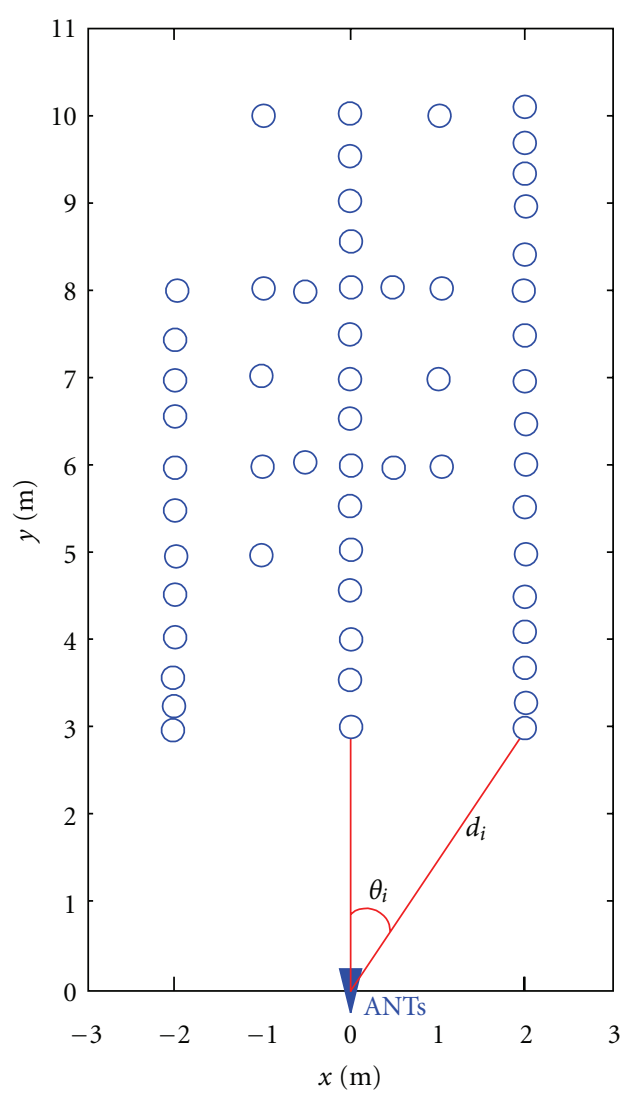

Figure 9: 57 locations of a target vehicle in outdoor experiments.

of the target vehicle are illustrated in Cartesian coordinates $(x, y)$ in Figure 9. Placing the radar at the origin of the center lane, the vehicle was parked at 57 locations between the left, center, and right lanes. The 57 locations were corresponded to a target direction with an azimuth angle $\theta_{i}$ between $-42^{\circ}$ and $+42^{\circ}$ and a target distance $d_{i}$ from $2.9 \mathrm{~m}$ to $10 \mathrm{~m}$ away from the origin, where $i=1, \ldots, 57$. The center of the rear bumper was used to define the target direction and distance, since the transmitted signals mainly impinged on the back of the target vehicle. The height of the radar antennas was 


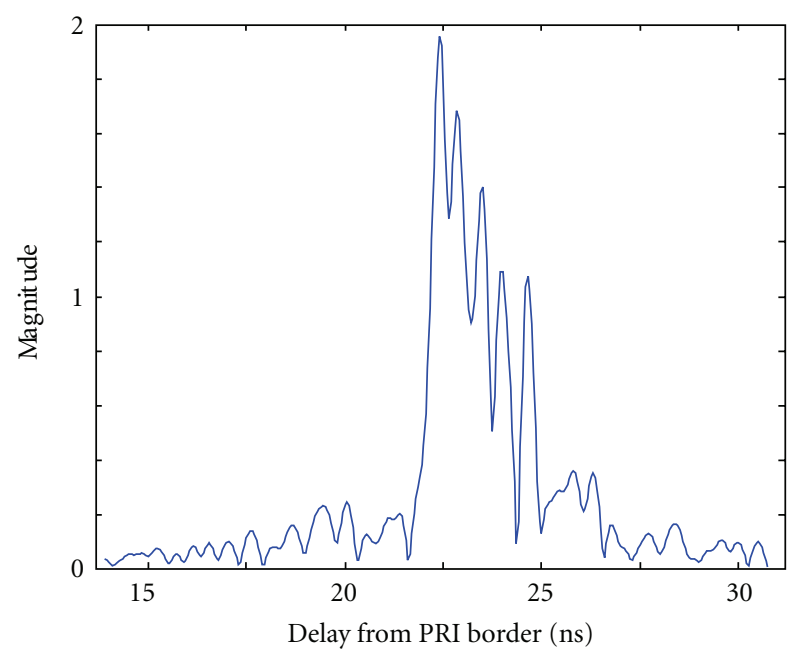

FIGURE 10: Pulse-compressed output signals from broadside in outdoor experiments.

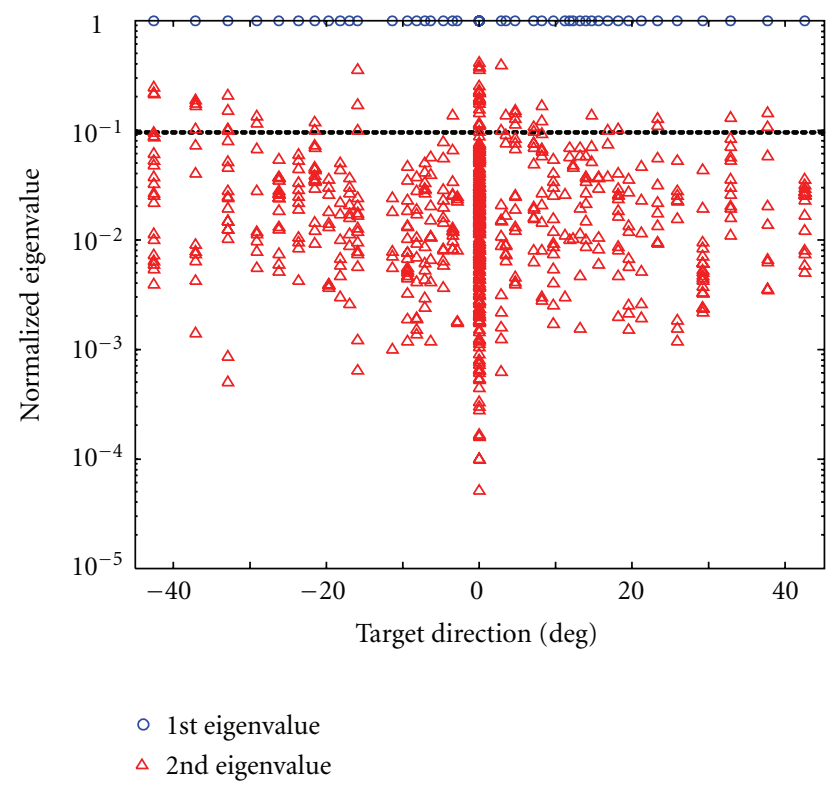

FIGURE 11: Normalized eigenvalue distribution derived from the measurements in outdoor experiments.

$0.6 \mathrm{~m}$, which was the same as that of the rear bumper. Effects of surrounding trees and buildings were negligible because the signals reflected from the vehicle were dominant in this setup.

Since there were no obstructions between the radar and the target vehicle, the channel model associated with this setup is line-of-sight (LOS) channel. Assuming a narrowband signal, the LOS path is given by a single propagation path because of low time resolution that is less than the inverse of the signal bandwidth. Whereas for the UWB signal, multipath components reflected from the target can be resolvable, so that every resolvable delay bin does not contain multipath components [3]. Moreover, the multipath components impinging on the antenna array are confined to small angle and delay corresponding to the target vehicle. This implies that the collected measurements are no longer treated as the specific channel models. We then characterize the UWB channel as the delay bin having either resolvable or nonresolvable multipath components, as described in the next section.

4.2. Resolvable/Nonresolvable Multipath Components. Multipath components were extracted for received signals by pulse compression given by (7). The obtained pulse-compressed output from the broadside and at a distance of $3.6 \mathrm{~m}$, namely, power delay profile, is shown in Figure 10. The resolvable multipath components were extracted to select the $K$ largest peaks as in (8) and then classified as three regions with respect to the lane of the parked vehicle: the left with minus angle, the center with the broadside, and the right with plus angle. The extracted number of multipath components was averaged over each region. The mean value on the broadside was the largest value at 5.1, while those in the left and right regions were, respectively, 3.0 and 4.1. This is because when the multipath components arriving from different directions have the same delay, the signal strength on the broadside is higher than that in the other regions, as shown in Figure 5. Therefore, the number of extracted peaks, depending on signal strength, decreases as the target direction moves away from the broadside.

Next, the nonresolvable multipath components within the extracted resolvable multipath components were evaluated. Setting $N_{\mathrm{smp}}=2$ and calculating the covariance matrix $\mathbf{R}$ as described in Section 2.2, two eigenvalues $\lambda_{1}$ and $\lambda_{2}$ were obtained by eigendecomposition of $\mathbf{R}$. The eigenvalue distribution normalized with $\lambda_{1}$ is illustrated in Figure 11. It is observed that some of eigenvalues $\lambda_{2}$ are larger than $10^{-1}$; the detection boundary was described in Section 3.2. Thus, the resolvable multipath component having $\lambda_{2}$ larger than $10^{-1}$ can be defined as a nonresolvable multipath component, as described in Section 2.2.

The number of nonresolvable components was also averaged in each region, and then the ratio of the number of nonresolvable components to that of resolvable components was calculated. The obtained ratios in the left region, on the broadside, and in the right region were $7.6 \%, 8.7 \%$, and $6.9 \%$, respectively. The nonresolvable multipath components are observed to concentrate on the broadside. This is because the multipath components, having a smaller delay than the monopulse duration of $1 \mathrm{~ns}$, tend to impinge on the receive array when the array is placed parallel to the four sides of the vehicle. In this experiment, a vehicle located on the broadside is parallel to the receive array, as shown in Figure 8.

4.3. AOA Estimation. The AOA was also estimated for each extracted peak using the MUSIC and the MVDR algorithms [8] used in the second experiment. The AOAs for the MUSIC versus the target direction are illustrated in Figure 12. A similar plot is obtained by MVDR algorithm. The two regression curves calculated from the anechoic chamber experiments are also plotted by solid lines. Note that the AOAs from the right region have an offset from the regression 


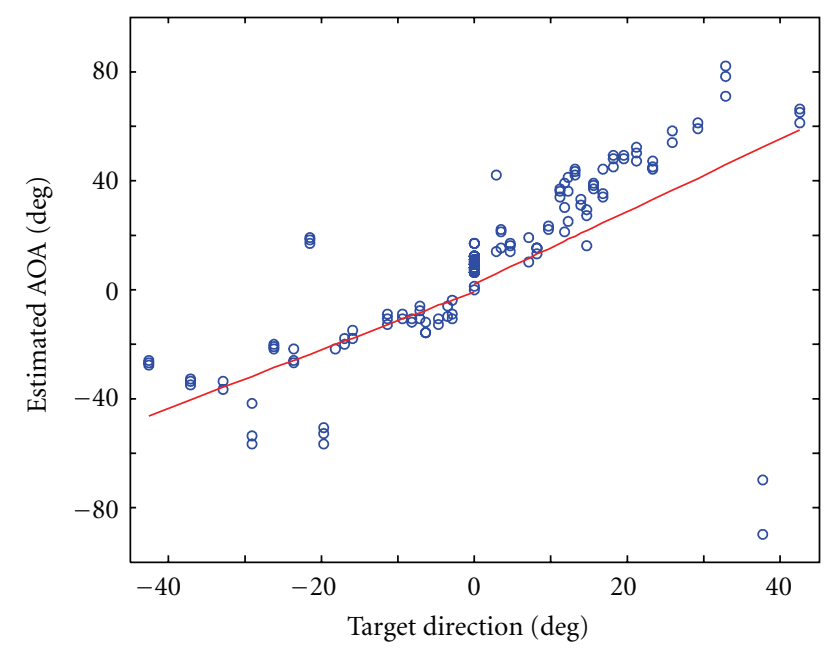

FIGURE 12: Estimated AOA by MUSIC derived from the measurements in outdoor experiments.

curve; this may be attributed to antenna misalignment. It can be seen that the AOAs from the target direction between $-20^{\circ}$ and $+20^{\circ}$ are close to the regression curves. Because of the resolution limitation caused by the two-element array and the anisotropic antenna radiation pattern, the number of outliers increases as the target angle moves away from the broadside.

4.4. Ranging and Multipath Spread. A UWB signal enables precision ranging capabilities because of a time resolution on the order of $1 \mathrm{~ns}$ [23]. The ranging of each location is obtained by finding the largest peak in the measured power delay profile. This approach is similar to a threshold-based energy detector-based time-of-arrival estimator [7] because a preamble structure resembles the transmitted pulse trains as shown in Figure 1. Since the delay from the PRI boundary to the largest peak is round-trip time between the origin and the target vehicle, the ranging is obtained from half of the delay time, as given by

$$
\hat{d}_{i}=\frac{c T_{i}}{2}, \quad i=1,2, \ldots, 57,
$$

where $c$ is the light velocity and $T_{i}$ is the delay bin of the $i$ th location. Each reference distance between the origin and the $i$ th location was measured from the receive array to the center of the vehicle on the rear bumper. The standard deviation of the ranging errors at each location versus the target distance is shown in Figure 13. The ranging resolution of $0.15 \mathrm{~m}$ corresponding to the monopulse duration of $1 \mathrm{~ns}$ is also plotted in a solid line. A single outlier more than the ranging resolution of $0.15 \mathrm{~m}$ is observed regardless of the shortest target distance. This phenomenon is also explained by the magnitude of extracted multipath component, as shown in Figure 5. Since the magnitude decreased as the target direction moved away from the broadside, the multipath component corresponding to the rear bumper center could not be precisely extracted at the location with the largest target direction. In other words, the largest peak of measured

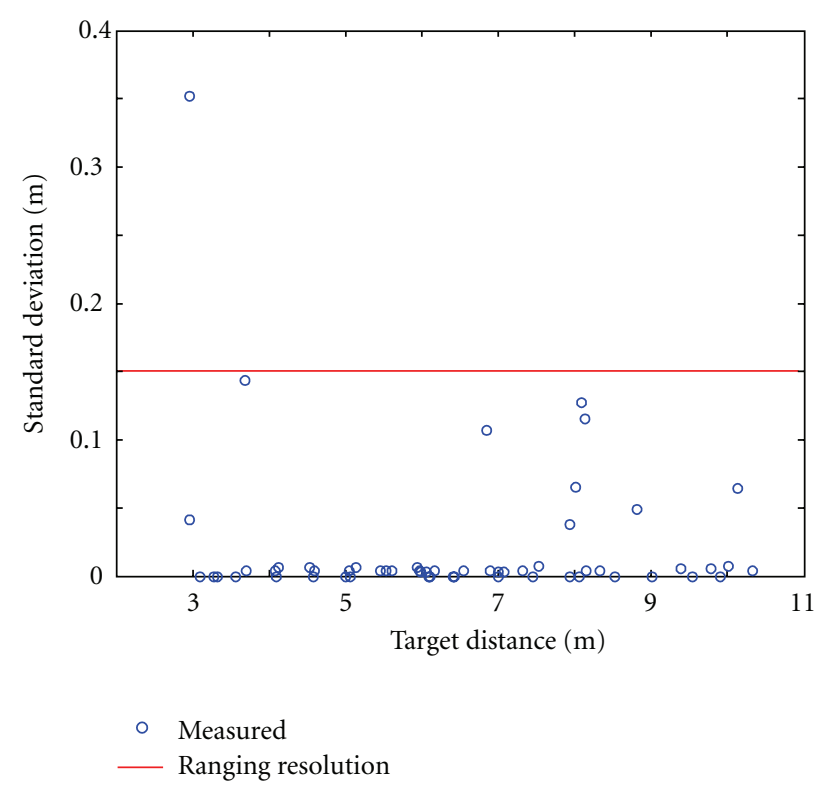

Figure 13: Standard deviation of ranging errors in outdoor experiments.

power delay profile did not always arrive from the rear bumper center when the target vehicle moved away from the broadside.

Thus, it seems intuitive that the obtained power delay profile from the target vehicle contains mostly reflected path components around the rear bumper center. We next estimate the propagation paths arriving from the target vehicle, and then define a multipath spread of the profile as a difference between the shortest and the largest arrival distances of multipath components to estimate the reflection point corresponding to each multipath component. The multipath spread of each target location is a plotted 3D graph, as shown in Figure 14. The resulting spread on the broadside ranges from 0.29 to $0.5[\mathrm{~m}]$, so the distance is too long to generate the power delay profile from only directly reflected path components from the rear bumper. Therefore, from the rear surface of the vehicle shown in Figure 8 , the reflection points of multipath components may include the pillar and roof as well as the bumper and the ground reflection path as well as the direct path. On the other hand, the multipath spread in the left and right regions tend to decrease as the target distance increases and target angle moves away from the broadside. This is mainly due to two reasons. First, since the side of the vehicle enables to contribute the reflection, the width of probable reflection surface increases as target angle moves away from the broadside. Second, the width of probable reflection surface on the vehicle decreases in proportion to distance and then more than one multipath component with almost the same delay tends to fall in a single peak.

4.5. Effect of Number of Averaging PRIs. The analyses so far has used the measurements with the averaging of $10^{4}$ PRIs and a sampling rate of 20 Gsps. The number of PRIs is an 


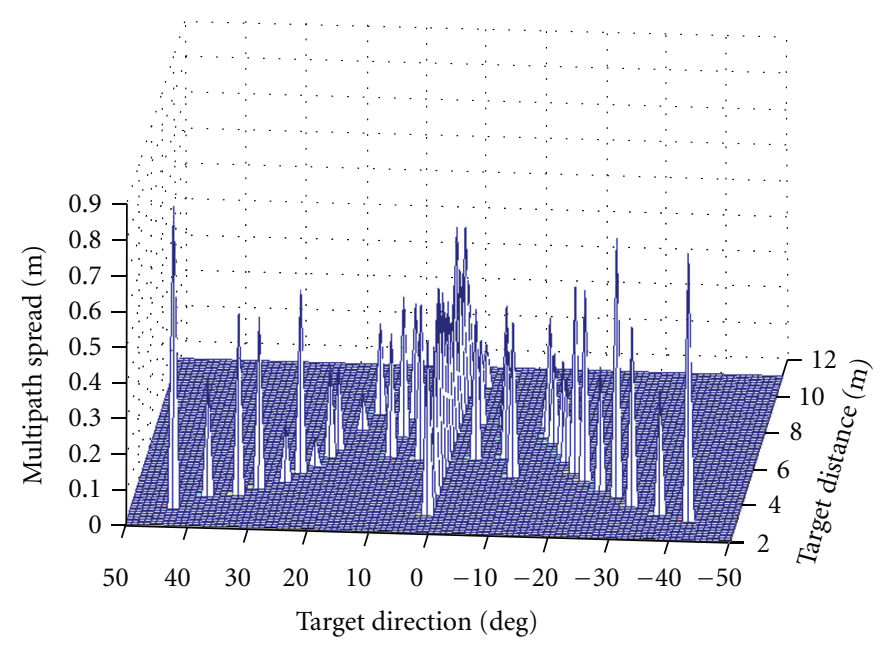

FIgURE 14: Multipath spread from a target vehicle in outdoor experiments.

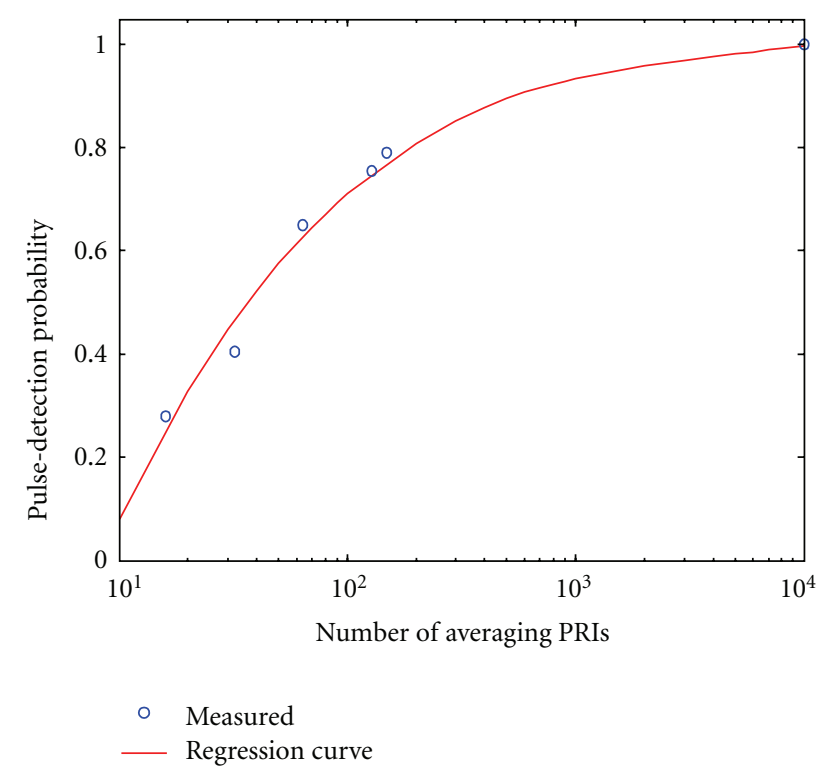

FIGURE 15: Pulse-detection probability versus number of averaging PRIs in outdoor experiments.

important parameter to improve accuracy of practical radar systems. The signals of each PRI were also recorded on the DSO with a sampling rate of 10 Gsps at the locations, as shown in Figure 9. The recorded signals were averaged over $N_{\text {ave }}$ PRIs, where $N_{\text {ave }}=16,32,64,128$, and 149 , since the maximum number of PRIs was 149 owing to the limitation of memory size on the DSO with $10 \mathrm{Gsps}$ sampling. Note that when calculating $\mathbf{R}, N_{\text {smp }}=1$ (total 3 samples) was selected for $10 \mathrm{Gsps}$ sampling.

For the measurements of 20 Gsps sampling, the ranging accuracy was verified as described in Section 4.4. We then define a ratio of correctly estimated ranging at each location as a pulse-detection probability. For example, the pulsedetection probability for averaging of $10^{4}$ PRIs was unity, since the estimated ranging error was zero. The resulting pulse-detection probability versus the averaging $N_{\text {ave }}$ is plotted in Figure 15. The regression curve is also plotted. The probability increases with $N_{\text {ave }}$ because of improvement of signal-to-noise ratio by averaging. The AOAs were next evaluated using only the multipath components corresponding to the correctly estimated ranging. However, almost all AOAs are not accurately estimated for $N_{\text {ave }}$ less than or equal to 128 . These results show that the level of the extracted peak after the pulse compression is generally below that of noise and thus; time average cannot converge to the true mean value.

\section{Conclusions}

This paper has presented the multipath resolution and direction findings of a UWB monopulse radar equipped with a two-element array. We cannot perform more detailed analyses owing to experimental limitations, such as the number of array elements, the number of monopulses per PRI, the received monopulse distortion, and measuring in static environment only. However, since the obtained results are useful parameters for performance analysis by computer simulation, a more practical UWB monopulse radar with multiple-element array will be designed. Moreover, the proposed estimation methods for resolvable/nonresolvable multipath components will be a valuable tool for any other UWB monopulse radars. The number of averaging monopulses is an important parameter for accuracy of ranging and direction finding. However, the observations must be corrected in static or quasistatic environments. Therefore, for practical use, a number of monopulses per PRI, sampling rate, and multiple-element array must be considered to increase the number of observations as quickly and accurately as possible.

\section{Acknowledgment}

This paper was supported in part by Grant-in-Aid for Scientific Research (C) KAKENHI 22560430. 


\section{References}

[1] I. Gresham, A. Jenkins, R. Egri et al., "Ultra-wideband radar sensors for short-range vehicular applications," IEEE Transactions on Microwave Theory and Techniques, vol. 52, no. 9, pp. 2105-2122, 2004.

[2] T. Kobayashi, N. Takahashi, M. Yoshikawa, K. Tsunoda, and N. Tenno, "Measurement of automobile UWB radar cross sections at Ka band," in Ultra-Wideband, Short-Pulse Electromagnetics 7, F. Sabath, E. L. Mokole, U. Schenk, and D. Nitsch, Eds., pp. 586-592, Springer, New York, NY, USA, 2007.

[3] A. F. Molisch, "Ultra-wide-band propagation channels," Proceedings of the IEEE, vol. 97, no. 2, pp. 353-371, 2009.

[4] Q. T. Zhang and S. H. Song, "Parsimonious correlated nonstationary models for real baseband UWB data," IEEE Transactions on Vehicular Technology, vol. 54, no. 2, pp. 447455, 2005.

[5] R. C. Qiu, "A study of the ultra-wideband wireless propagation channel and optimum UWB receiver design," IEEE Journal on Selected Areas in Communications, vol. 20, no. 9, pp. 16281637, 2002.

[6] J. Adeane, W. Q. Malik, I. J. Wassell, and D. J. Edwards, "Simple correlated channel model for ultrawideband multiple-input multiple-output systems," IET Microwaves, Antennas and Propagation, vol. 1, no. 6, pp. 1177-1181, 2007.

[7] D. Dardari, A. Conti, U. Ferner, A. Giorgetti, and M. Z. Win, "Ranging with ultrawide bandwidth signals in multipath environments," Proceedings of the IEEE, vol. 97, no. 2, pp. 404425, 2009.

[8] S. H. Song and Q. T. Zhang, "Multi-dimensional detector for UWB ranging systems in dense multipath environments," IEEE Transactions on Wireless Communications, vol. 7, no. 1, pp. 175-183, 2008.

[9] P. A. Bello, "Evaluation of mobile ultra wideband modems in dense multipath-part 1: channel model," IEEE Transactions on Wireless Communications, vol. 6, no. 11, pp. 4145-4153, 2007.

[10] T. Santos, F. Tufvesson, and A. Molisch, "Modeling the ultra-wideband outdoor channel: model specification and validation," IEEE Transactions on Wireless Communications, vol. 9, no. 6, pp. 1987-1997, 2010.

[11] J. Choi, N. G. Kang, Y. S. Sung, and S. C. Kim, "Empirical ultra wide band channel model for short range outdoor environments," in 65th Vehicular Technology Conference (VTC '07), pp. 1579-1583, April 2007.

[12] J. Y. Lee, "UWB channel modeling in roadway and indoor parking environments," IEEE Transactions on Vehicular Technology, vol. 59, no. 7, pp. 3171-3180, 2010.

[13] H. Enkoji, N. Hashimoto, H. Aoki, N. Iwakiri, and T. Kobayashi, "Evaluation of a UWB phase-comparison monopulse short-range radar," in International Conference on Space, Aeronautical and Navigational Electronics (ICSANE '10), October 2010 .

[14] H. Krim and M. Viberg, "Two decades of array signal processing research: the parametric approach," IEEE Signal Processing Magazine, vol. 13, no. 4, pp. 67-94, 1996.

[15] B. R. Mahafza, Radar Systems Analysis and Design Using MATLAB, Chapman \& Hall/CRC, New York, NY, USA, 2005.

[16] P. Withington, "Impulse radio overview," Time Domain Corp., http://user.it.uu.se/ carle/Notes/UWB.pdf.

[17] X. Luo, L. Yang, and G. B. Giannakis, "Designing optimal pulse-shapers for ultra-wideband radios," Journal of Communications and Networks, vol. 5, no. 4, pp. 344-353, 2003.
[18] R. Xu, Y. Jin, and C. Nguyen, "Power-efficient switchingbased CMOS UWB transmitters for UWB communications and radar systems," IEEE Transactions on Microwave Theory and Techniques, vol. 54, no. 8, pp. 3271-3277, 2006.

[19] R. G. Bosisio, Y. Y. Zhao, X. Y. Xu et al., "New wave radio," IEEE Microwave Magazine, vol. 9, no. 1, pp. 89-100, 2008.

[20] M. G. M. Hussain, "Principles of space-time array processing for ultrawide-band impulse radar and radio communications," IEEE Transactions on Vehicular Technology, vol. 51, no. 3, pp. 393-403, 2002.

[21] L. Sakkila, A. Rivenq, C. Tatkeu, Y. El Hillali, J. P. Ghys, and J. Rouvaen, "Methods of target recognition for UWB radar," in IEEE Intelligent Vehicles Symposium (IV'10), pp. 949-954, San Diego, Calif, USA, June 2010.

[22] S. Gezici and H. V. Poor, "Position estimation via ultra-wideband signals," Proceedings of the IEEE, vol. 97, no. 2, pp. 386403, 2009.

[23] R. J. M. Cramer, R. A. Scholtz, and M. Z. Win, "Evaluation of an ultra-wide-band propagation channel," IEEE Transactions on Antennas and Propagation, vol. 50, no. 5, pp. 561-570, 2002. 

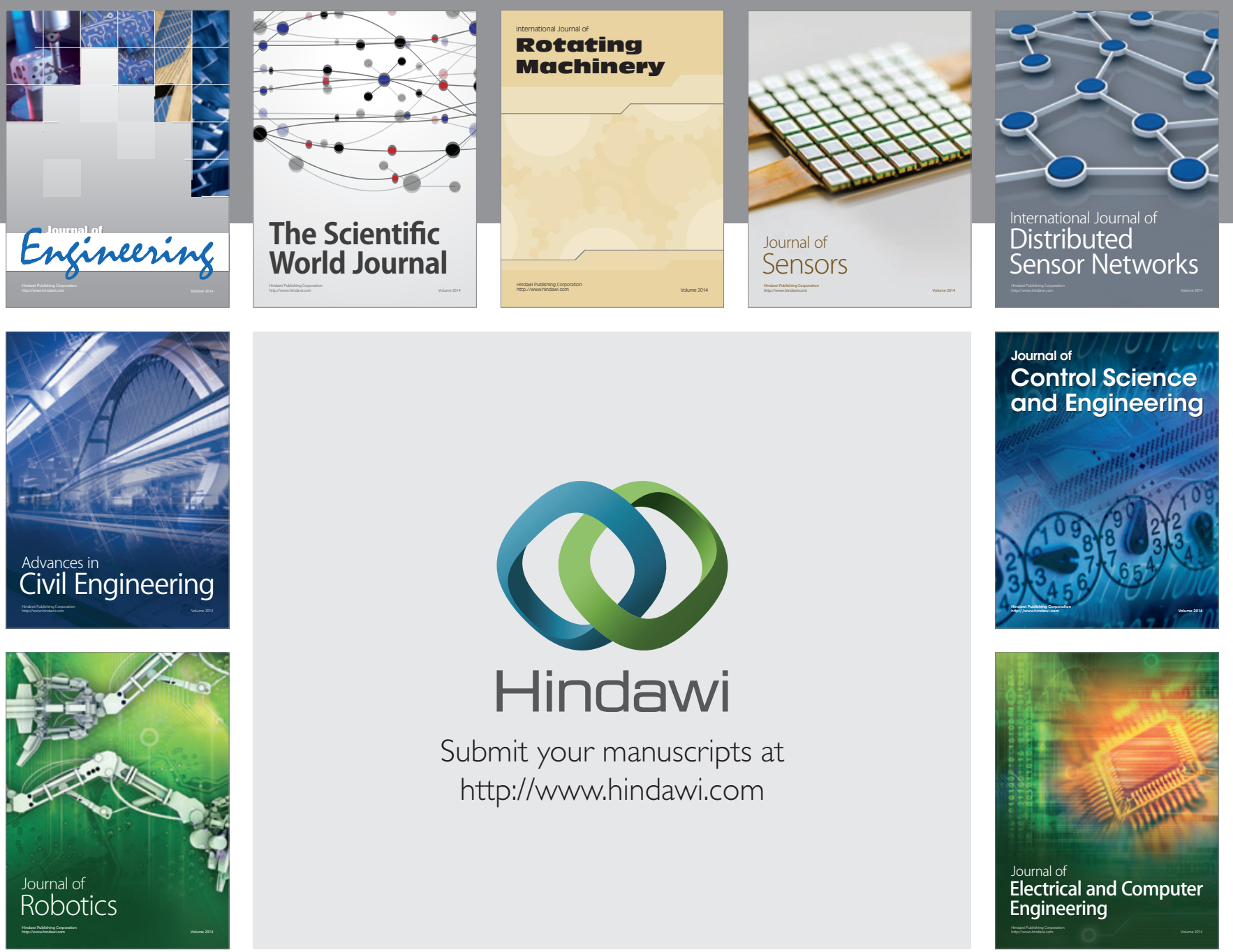

Submit your manuscripts at

http://www.hindawi.com
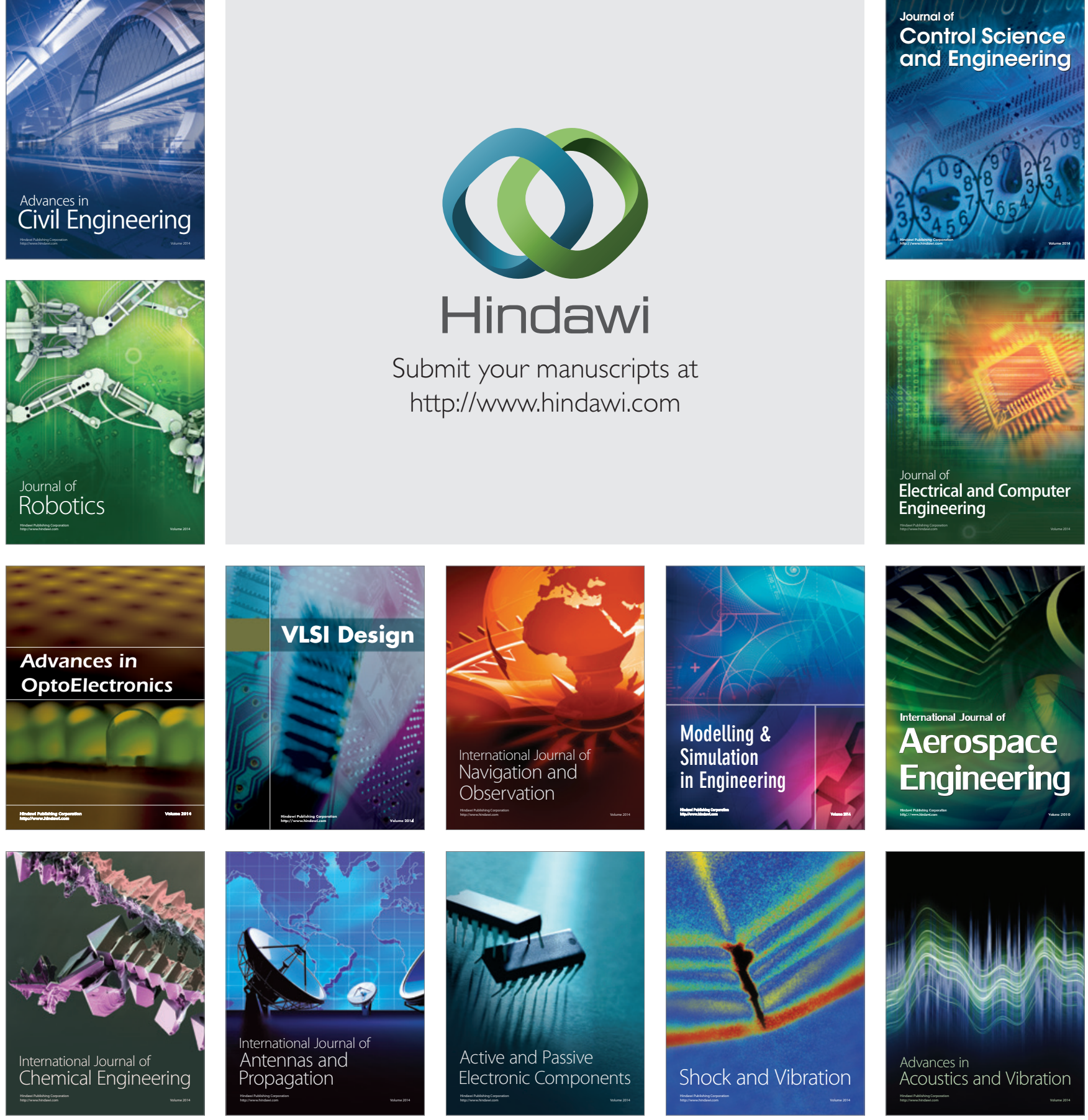